\title{
Vagus-Preserving Proximal Gastrectomy with Double- Flap Technique for Gastrointestinal Stromal Tumor involving the Esophagogastric JunctionखA Case Report
}

\section{Wei Peng ( $\sim$ pengwei_11636@126.com )}

Second Affiliated Hospital of Soochow University https://orcid.org/0000-0002-6454-1223

Yi-Kai Huang

Second Affiliated Hospital of Soochow University

\section{Yong-You Wu}

Second Affiliated Hospital of Soochow University

\section{Wei Gong}

Second Affiliated Hospital of Soochow University

\section{Zheng-Rong Chen}

Second Affiliated Hospital of Soochow University

\section{Qiang Chen}

Second Affiliated Hospital of Soochow University

\section{Wen-Zhao Su}

Second Affiliated Hospital of Soochow University

\section{Ming Cheng}

Second Affiliated Hospital of Soochow University

\section{Research Article}

Keywords: Gastrointestinal stromal tumors, Vagus-preserving proximal gastrectomy, Double-flap technique, Esophagogastrostomy, Esophagogastric junction, Case report

Posted Date: August 23rd, 2021

DOl: https://doi.org/10.21203/rs.3.rs-779253/v1

License: (1) This work is licensed under a Creative Commons Attribution 4.0 International License. Read Full License 


\section{Abstract}

Background: Gastrointestinal stromal tumor (GIST) involving the esophagogastric junction (EGJ) is a relatively rare but thorny problem. It represents a dilemma between complete resection and impairment of quality of life (QOL) for loss of cardiac sphincter or even the whole stomach. Thus, emergence of function preserving gastrectomy is necessary and may provide partial solution to this hot potato. Here, we would like to introduce a method which successfully settle this matter through a clinical case.

Case presentation: A 46-year-old male complaining of a progressive dysphagia. A huge GIST with 120mm in diameter was diagnosed by enhanced CT scan and pathological exam. Imatinib mesylate (IM) was initially prescribed, the tumor was shrank to a large extent while drug resistance emerged 6 months after therapy, leaving a mass approximately $90 \mathrm{~mm}$ in diameter. We applied a method of vagus preserving proximal gastrectomy (VP-PG) and esophagogastrostomy with double-flap technique (DFT) for the patient. The operation was smooth and postoperative courses within a 12-months follow-up were uneventful. The patient had no digestive symptoms but slight leucopenia due to IM toxicity during this medication period.

Conclusion: We consider that the application of VP-PG and DFT subsequent to neoadjuvant therapy with IM is likely to be an effective and feasible option for GISTs with EGJ involvement.

\section{Background}

GIST, originates from interstitial cells of Cajal, is the most common mesenchymal tumors in digestive tract with $50-60 \%$ of which located at the stomach ${ }^{[1]}$, especially at the upper third site. Preoperative targeted therapy with IM is recommended for initially unresectable GISTs or those invading important structures ${ }^{[2]}$, while surgical resection is inevitable in most cases, leading damage of important structures and impairment of QOL. GISTs located at the EGJ represents a clinical challenge. Previous method of total gastrectomy may cause life-time nutritional problems while PG with tradition esophagogastrostomy may induce severe reflux esophagitis. Moreover, routine gastrectomy with truncal vagotomy increases the occurrence of gallstones and diarrhea. However, lymphadenectomy is unnecessary for GISTs due to its low incidence of lymph node metastasis ${ }^{[3]}$, which makes it feasible for vagus preservation during the operation. Thus, the sequence of neoadjuvant therapy and subsequent PG with vagus preservation and anti-reflux technique seems an ideal and promising method for GISTs with EGJ involvement. Here, we present a case successfully managed with this strategy.

\section{Case Presentation}

A 46-year-old male, $59 \mathrm{~kg}$ in weight, complaining of a progressive dysphagia for nearly 3 months, was referred to our department of Gastrointestinal surgery. A large lesion located at the EGJ, about $120 \mathrm{~mm}$ in diameter, was observed during CT scan. Endoscopic ultrasonography (EUS) identified a mixed echo mass within the submucosal layer. Immunochemistry of biopsy specimens demonstrated a CD34(+) and 
CD117(+) phenotype, leading to a definitive diagnosis of GIST. Initially, the patient received preoperative IM treatment with a dose of $400 \mathrm{mg} /$ day recommended by a previous research ${ }^{[4]}$ but was finally reduced to $300 \mathrm{mg} /$ day due to severe leukopenia. The tumor was shrank remarkably during the beginning 4-month of therapy while drug resistance developed after 6 months, leaving a mass approximately $90 \mathrm{~mm}$ in diameter (Fig. 1). Surgical intervention was attempted after obtaining informed consent. Laparoscopic procedure for GISTs with an extremely large size and a special location was excluded due to its uncertainty of oncological safety. Laparotomy was made by with a midline incision. No peritoneal dissemination, nodal enlargement or hepatic metastasis was observed during intra-abdominal exploration. We operated carefully to detect and free the anterior and posterior trunks of the vagus nerve. The anterior gastric branches of the vagus were transected below the hepatic branch, just preserve the anterior trunk and its hepatic branch intact (Fig. 2). Then, gastric mesentery was divided, the posterior trunk and its celiac branch were preserved. The esophagus was transected $2 \mathrm{~cm}$ above the mass and the tumor was removed with a preservation of distal 2/3 gastric tissues (Fig. 3). Reconstruction with doubleflap technique (DFT) was then performed on the remnant stomach as described by Kuroda et al ${ }^{[5]}$. Briefly, $\llbracket$ An $\mathrm{H}$-shaped $2.5 \times 3.5 \mathrm{~cm}$ seromuscular double-flap was created. $\otimes T$ The gastric mucosa was opened for the anastomosis. $\bigotimes$ The posterior side of the esophagus was fixed to the gastric remnant at the upper edge of the flap. 区Anastomosis of the esophagus and the remnant stomach was created by hand-sewn. $\triangle T$ The flaps were positioned to cover the anastomosis site in a Y-shape (Fig. 4).

The operation was smooth. Oral intake of liquid was resumed 3 days after surgery. The patient was allowed to be discharged on the 8th postoperative day with a body weight of $51 \mathrm{~kg}$, complaining of no digestive discomforts. Histopathological immunohistochemistry confirmed an activation of KIT, positivity of CD117, CD34 and Dog1. Two weeks after operation, barium meal X-ray was preserved, indicating a slight anastomotic stricture with no leakage or bleeding (Fig. 5), even though the patient complained of no dysphagia and then restarted IM medication with a same dose as before. During the adjuvant targeted therapy, the patient still suffered from leukopenia owning to IM toxicity. Accordingly, Leucogen tablets with a dose of $60 \mathrm{mg}$ t.i.d was prescribed and the leukocyte counts were able to maintain within an average level of $2.4^{\star} 10^{\wedge} 9 / \mathrm{L}$. Gastroscopy was conducted on the 5 th postoperative month, revealing a good-shaped pseudo gastric fundus with no severe anastomotic complications but a little erosion closed to the anastomosis (Fig. 6). Nevertheless, the patient had no gastroesphageal reflux symptoms, like heartburn, acid regurgitation, as well as other digestive discomforts. We described the patient as Grade $\mathrm{A}$ according to the Los Angeles classification ${ }^{[6]}$. PPI was prescribed after the exam. Furthermore, a gallbladder contraction text was applied to assess the activity of vagus nerve after 6 month, the volume of gallbladder lessened by $48 \%$ before and after the fat mile which demonstrated a normal contractile function. After 9 months of follow-up, the patient regained weight to $59 \mathrm{~kg}$ with normal hemoglobin and total protein concentrations. Recently, the patient returned to our department for his 12-months recheck. Enhanced CT scan was provided, indicating a normal structure of reconstructed EGJ and there were no signs of relapse of the tumor around the gastric area.

\section{Discussion}




\section{Vagus functions}

Vagus nerve plays an important role in the functional integrality of abdominal organs. It had been previously reported by Uyama et al. that the heptic branch, correlating with the function of liver and biliary system, facilitating bile excretion and preventing gallstone formation ${ }^{[7]}$. Moreover, an intraoperative neurophysiologic testing observed contractile movement near the duodenum following a stimulation of

the hepatic branch ${ }^{[8]}$. A rodent study demonstrated that the hepatic branch served as vagal afferent nerve for interoception, collecting information from peripheral organs, activating different regions of the brain depending on the nature of stimulus on rats ${ }^{[9]}$. The celiac branch is known to be associated with the motility of upper gastrointestinal tract. Responses of the duodenum and proximal jejunum $20 \mathrm{~cm}$ from Treitz ligament could be detected after stimulating the celiac branch according to the previous neurophysiologic testing. Furthermore, gastrointestinal hormone level is regulated by the vagus. Takiguchi et al ${ }^{[10]}$ observed a reduction level of postprandial ghrelin on postoperative day 7 after vaguspreserving gastrectomy, which was considered necessary for maintaining homeostasis and controlling energy balance. A study identified a less increase of Peptide YY3-36, a satiety signal related to inhibition of food intake in human after preserving the vagus nerve, preventing excessive body weight loss after gastrectomy ${ }^{[11]}$. In addition, vagus nerve could activate cholinergic anti-inflammatory pathway, reducing the occurrence of postoperative acute inflammation ${ }^{[12]}$. Although vagus preservation has been reported in pylorus preserving gastrectomy for distal gastric cancer in abundance, its preservation in proximal gastrectomy is rarely reported potentially due to its difficulty in manipulation and inadequate recognition of vagus functions. The value of vagus preservation, especially that of the celiac trunk, still needs to be intensively investigated. For gastric cancer, some surgeons may suspect the completeness of lymph nodes dissection. But for GISTs, it is oncologically safe, technically feasible and potentially beneficial which deserves more attention and attempt.

\section{Double-Flap technique}

Esophagogastrostomy $(E G)$ is a preferred reconstruction method for its reduced anastomosis and restoration of physiological passage of the digestive tract. However, a major concern for EG is reflux esophagitis for lose of cardia, which adversely affects patients' postoperative QOL. To surmount this obstacle, several reconstruction methods were established on the basis of EG. Double flap technique (DFT), a modified EG procedure which was first mentioned by a Japanese surgeon named Kamikawa ${ }^{[13]}$, represents another anti-reflux technique. The anastomosis and lower part of the esophagus are covered with seromuscular flaps of the gastric remnant which works as an uni-directional valve against reflux. The feasibility and utility of which has been supported by evidence from a multicenter retrospective study ${ }^{[14]}$. The Japanese Gastric Cancer Guidelines recommends that PG should be applied for cT1N0M0 proximal gastric cancer less than $4 \mathrm{~cm}$ in diameter when at least half of the gastric tissues can be preserved $^{[15]}$. The prerequisite for DFT is to ensure a preservation of at least $2 / 3$ of the distal gastric remnant to maintain the function of the stomach according to domestic and international surgical 
specialists. GIST near or invading the EGJ seems a good indication for EG with DFT, for its absence of lymphatic dissemination and non-infiltrative nature.

\section{Conclusion}

We present the case for the purpose of illustrating a standardized treatment of a GIST with a large size invading the EGJ. For GISTs of this type, especially the lower esophageal sphincter cannot spared even after preoperative targeted therapy with IM, our strategy of applying both VP and DF technique to PG ought to be an ideal option, which provides a feasible solution to clinical setting.

\section{Abbreviations}

GIST

Gastrointestinal stromal tumors

EGJ

Esophagogastric junction

VP-PG

Vagus-preserving proximal gastrectomy

DFT

Double-flap technique

EG

Esophagogastrostomy

QOL

Quality of life

IM

Imatinib mesylate

\section{Declarations}

\section{Ethics approval and consent to participate}

Not applicable.

\section{Consent for publication}

Informed consents for publication has been signed.

\section{Availability of data and materials}

Data sharing is not applicable to this article as no datasets were generated or analysed during the current study. 
The authors declare that they have no competing interests.

\section{Funding}

Suzhou Introduction Project of Clinical Medical Expert Group (No: SZYJTD201804).

\section{Authors' contributions}

Wei Peng was the first author of this manuscript. Yi-Kai Huang was the co first author who collected information, designed figures as well as revised and modified the mansucript with the same contribution. Yong-You Wu modified and approved the final manuscript and was the corresponding author of the article. Wei Gong, Zheng-Rong Chen, Qiang Chen, Wen-Zhao Su and Ming Cheng revised the mansucript and gave instructive suggestions to the author.

\section{Acknowledgements}

We would like to thank colleagues at the department of gastrointestinal surgery in our hospital.

\section{References}

1. Dhruv J, Patel W, Lutfi P, Sweigert, et al. Adjuvant Systemic Therapy For Intermediate And Large Gastric Gastrointestinal Stromal Tumors (GISTs): Is There A Survival Benefit Following Margin Negative Surgical Resection? Am J Surg. 2020;219(3):436-9.

2. Fiore $M$, Palassini $E$, Fumagalli $E$, et al. Preoperative imatinib mesylate for unresectable or locally advanced primary gastrointestinal stromal tumors (GIST). Eur J Surg Oncol. 2009;35(7):739-45.

3. Miettinen M, Furlong M, Sarlomo-Rikala M,et al. Gastrointestinal stromal tumors, intramural leiomyomas, and leiomyosarcomas in the rectum and anus: a clinicopathologic, immunohistochemical, and molecular genetic study of 144 cases. Am J Surg Pathol. 2001;25(9):1121-33.

4. Demetri GD, Mehren MV, Blanke CD, et al. Efficacy and safety of imatinib mesylate in advanced gastrointestinal stromal tumors. N Engl J Med. 2002;347(7):472-80.

5. Kuroda S, Nishizaki M, Kikuchi S, et al. Double-flap technique as an antireflux procedure in esophagogastrostomy after proximal gastrectomy. J Am Coll Surg. 2016;223(2):e7-13.

6. Lundell LR, Dent J, Bennett JR, et al. Endoscopic assessment of oesophagitis: clinical and functional correlates and further validation of the Los Angeles classification. Gut. 1999;5(2):172-80.

7. Uyama I, Sakurai Y, Komori Y, et al. Laparoscopic gastrectomy with preservation of the vagus nerve accompanied by lymph node dissection for early gastric carcinoma. J Am Coll Surg. 2005;200:1405. 
8. Kong SH, Kim SM, Kim DG, et al. Intraoperative Neurophysiologic Testing of the Perigastric Vagus Nerve Branches to Evaluate Viability and Signals along Nerve Pathways during Gastrectomy. J Gastric Cancer. 2019;19(1):49-61.

9. Yamada D, Koppensteiner P, Odagiri S, et al. Common Hepatic Branch of Vagus Nerve-Dependent Expression of Immediate Early Genes in the Mouse Brain by Intraportal L-Arginine: Comparison with Cholecystokinin-8. Front Neurosci. 2017;28(11):366.

10. Takiguchi S, Hiura Y, Takahashi T,et al. Preservation of the Celiac Branch of the Vagus Nerve during Laparoscopy-assisted Distal Gastrectomy: Impact on Postprandial Changes in Ghrelin Secretion. World J Surg. 2013;37(9):2172-9.

11. Kim HH, Park Ml, Lee SH, et al. Effects of vagus nerve preservation and vagotomy on peptide $\mathrm{YY}$ and body weight after subtotal gastrectomy. World J Gastroenterol. 2012;18(30):4044-50.

12. Pavlov VA, Wang H, Czura CJ, et al. The cholinergic anti-inflammatory pathway: a missing link in neuroimmunomodulation. Mol Med. 2003;9(5-8):125-34.

13. Kamikawa Y, Kobayashi T, Kamiyama S, et al. A new procedure of esophagogastrostomy to prevent reflux following proximal gastrectomy. Shoukakigeka. 2001;24:1053-60.

14. Kuroda S, Choda Y, Otsuka S, et al. Multicenter retrospective study to evaluate the efficacy and safety of the double-flap technique as antireflux esophagogastrostomy after proximal gastrectomy (rDFLAP Study). Ann Gastroenterol Surg. 2018;3(1):96-103.

15. Japanese Gastric Cancer Association. Japanese gastric cancer treatment guidelines 2014 (ver. 4). Gastric Cancer. 2017;20(1):1-19.

\section{Figures}




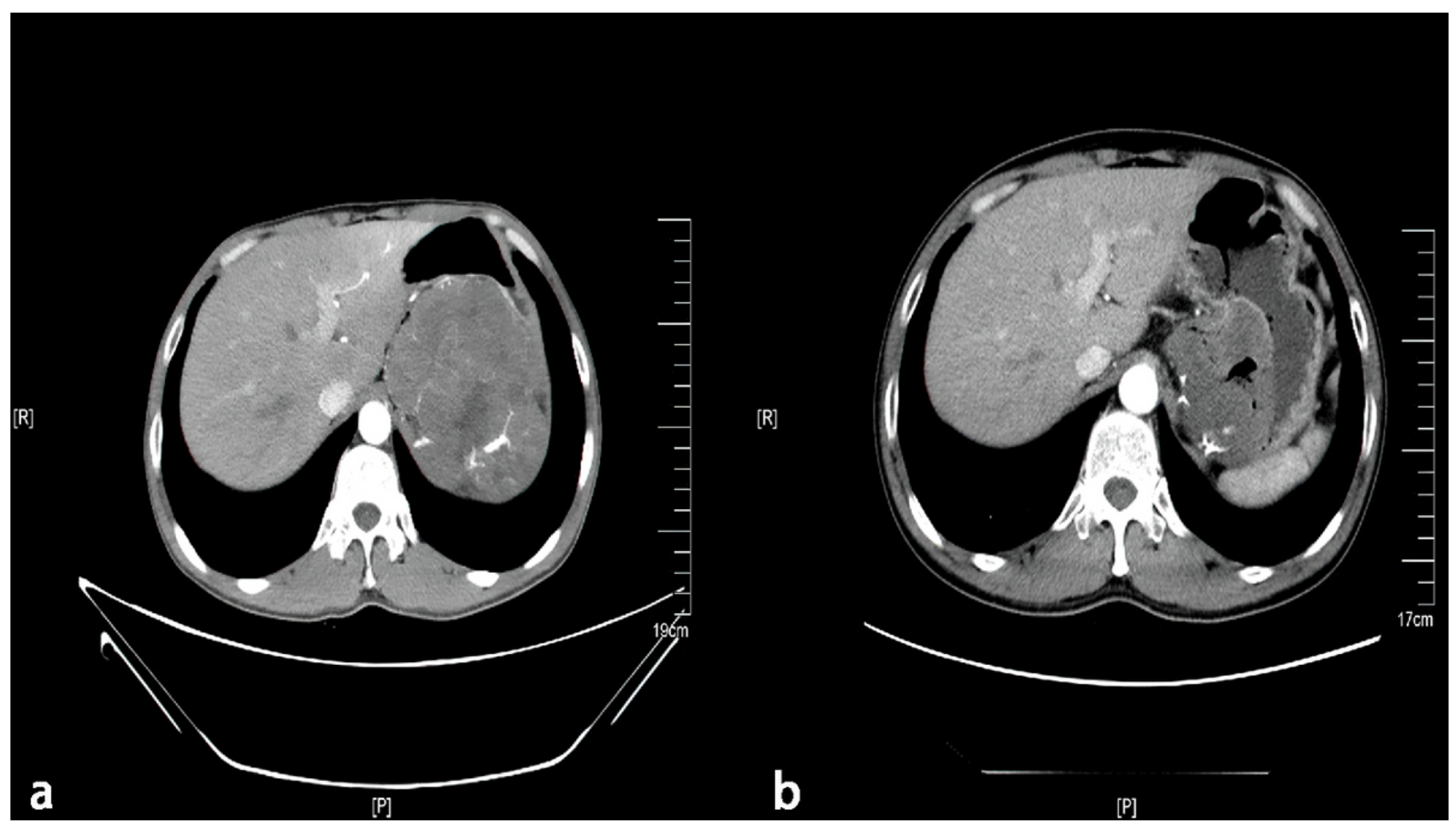

\section{Figure 1}

Abdominal enhanced CT scans represent clearly of the change of tumor size before and after the neoadjuvant therapy of IM. (Fig. a was the initial image compared with Fig. b which was the last image after 6-months of IM theraphy.) 


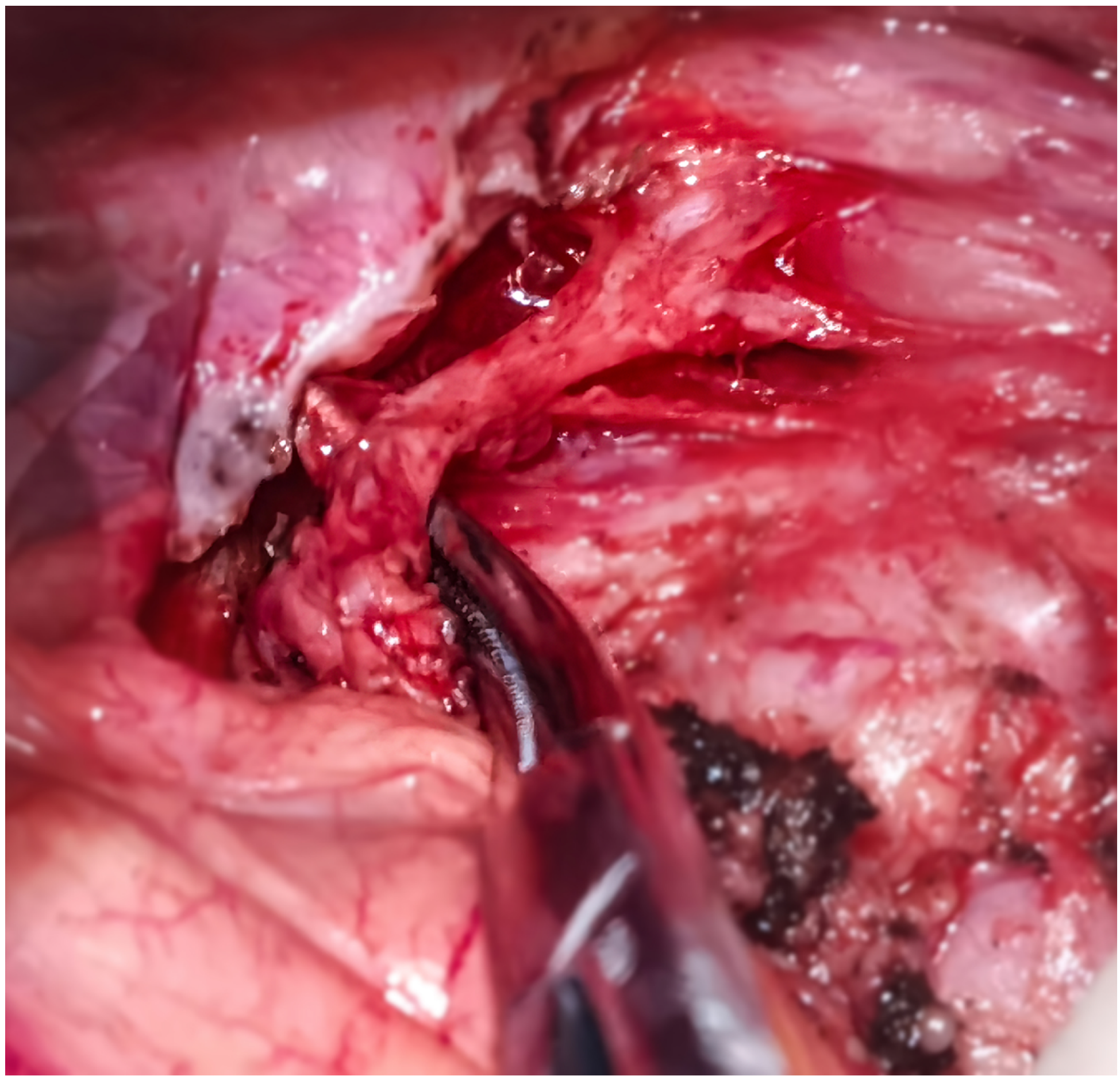

Figure 2

The anterior trunk of the vagus nerve was preserved during the procedure. 


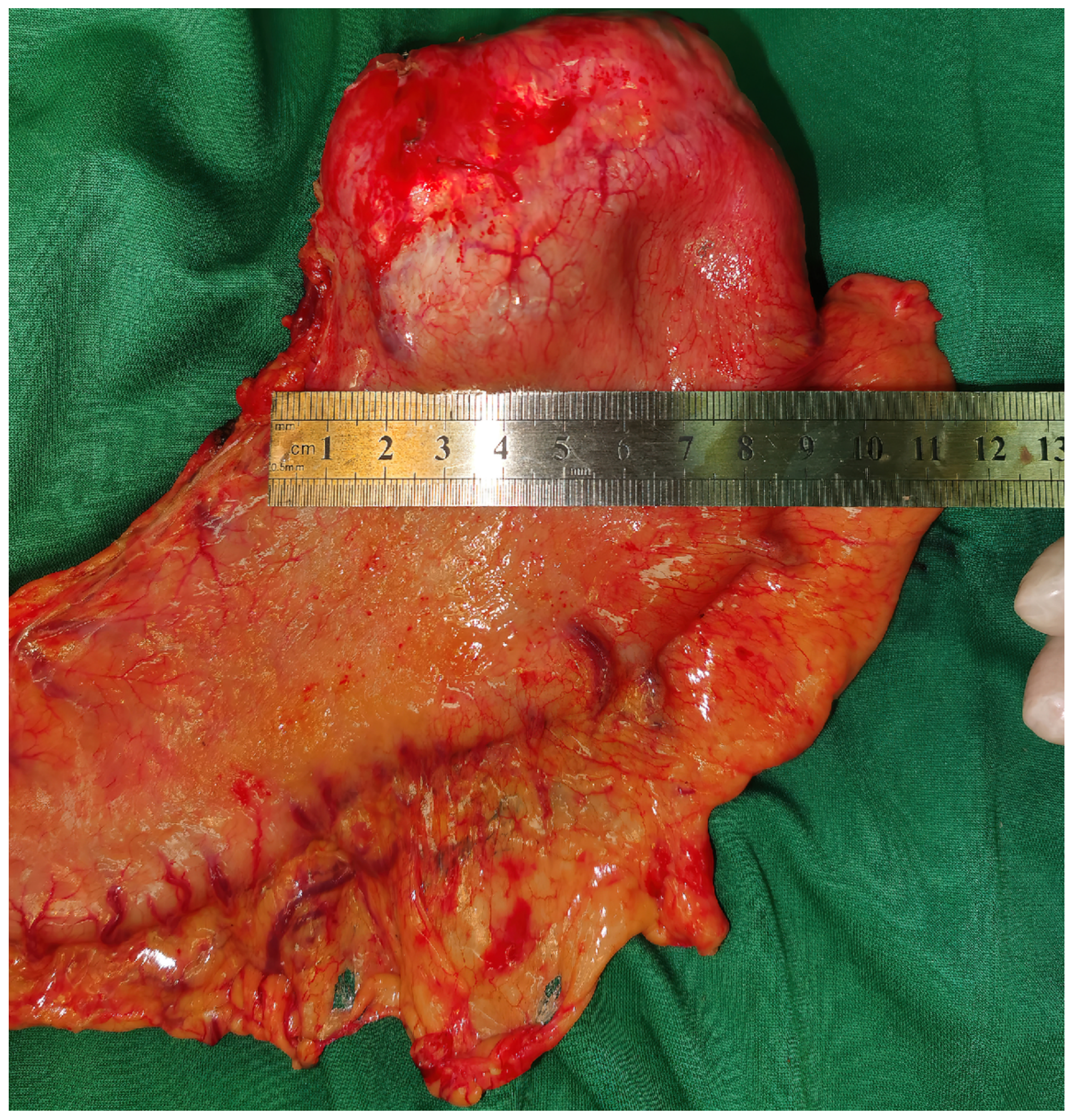

\section{Figure 3}

The tumor, approximately $90 \mathrm{~mm}$ in diameter, were pulled out of the abdominal cavity after transecting the esophagus with a linear stapler. 


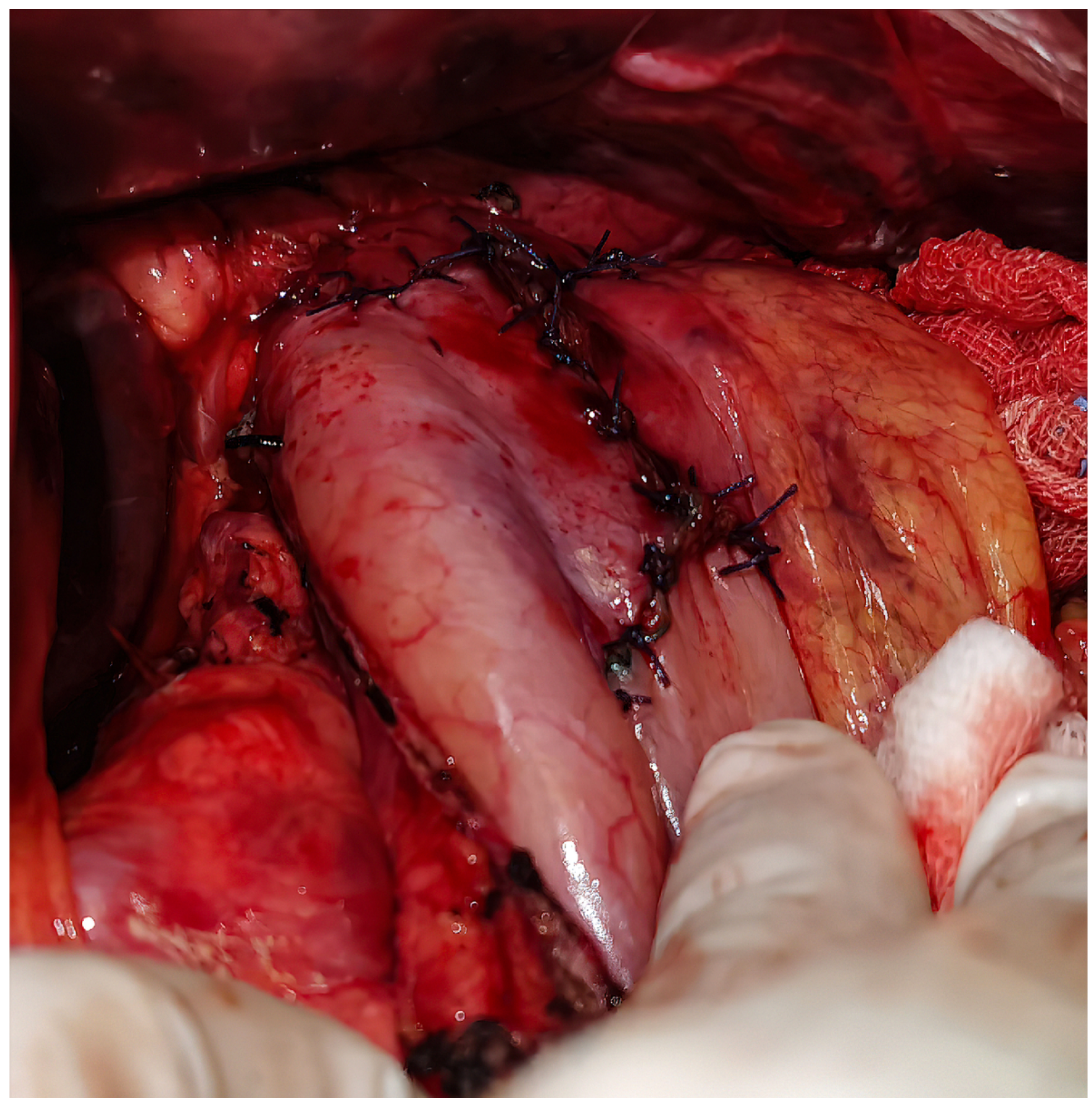

\section{Figure 4}

Double Flap Technique (DFT) was preformed during the procedure. Reconstruction was completed with both flaps covered on the anastomosis. 


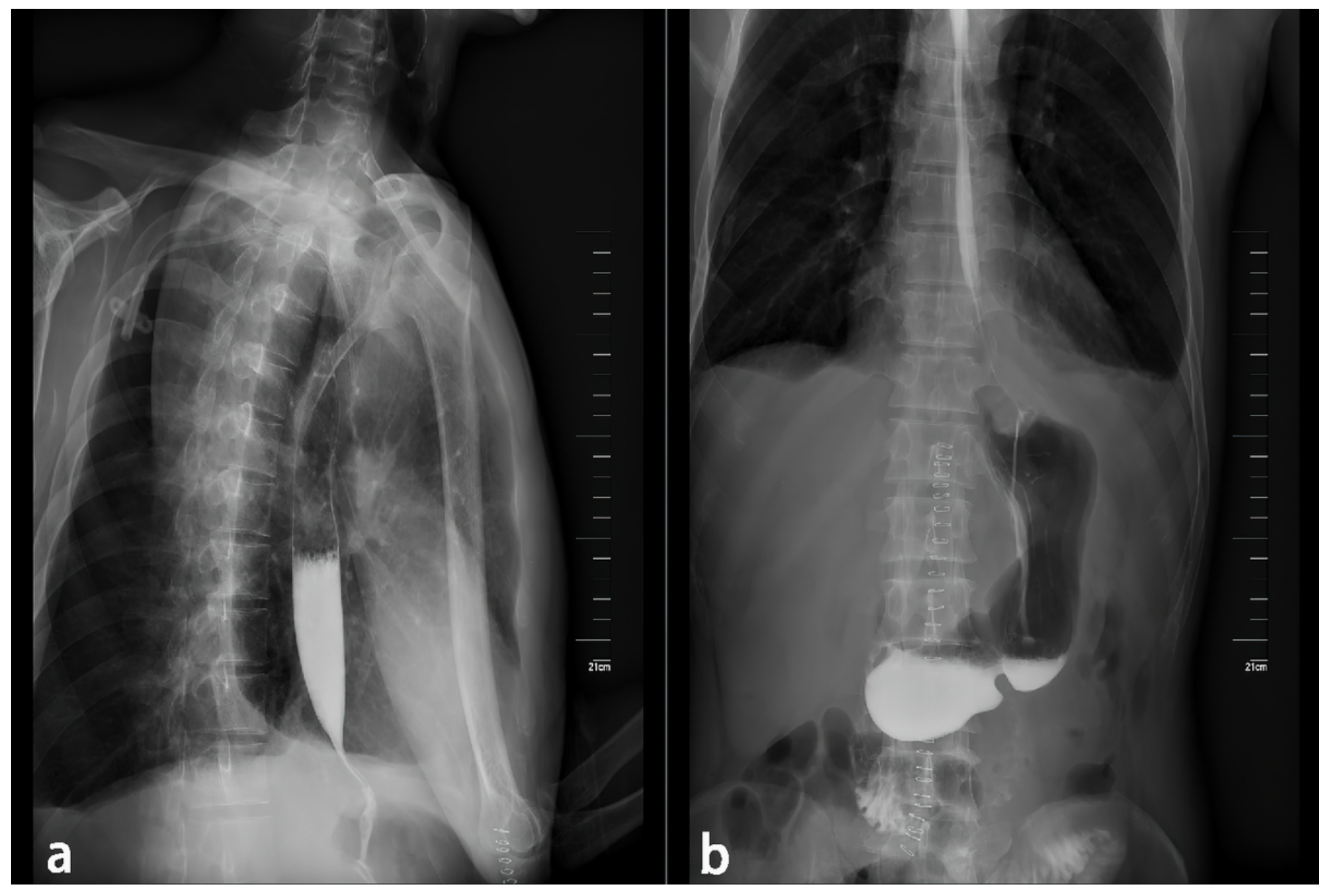

Figure 5

Barium meal X-ray was performed 2 weeks after the operation, indicating a slight anastomotic stricture, but no leakage or bleeding.

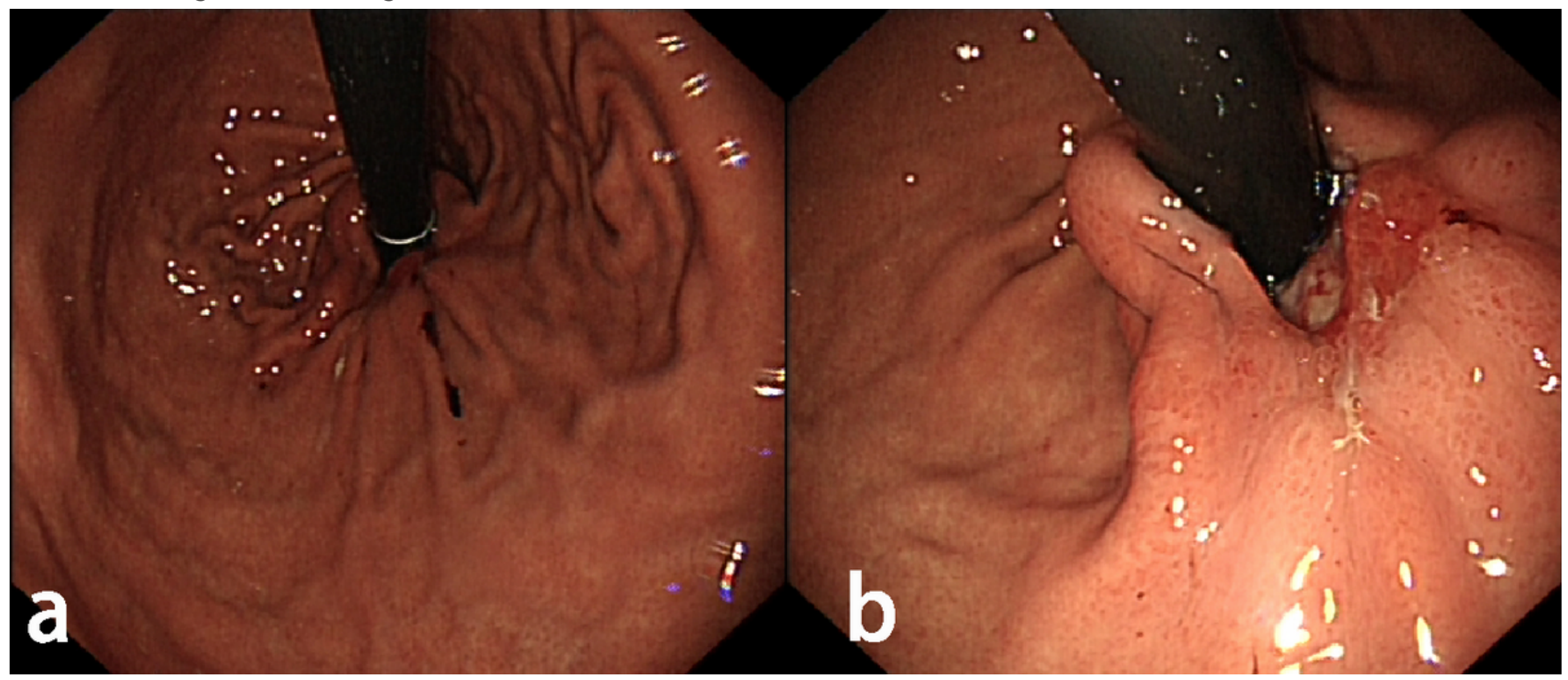


Figure 6

Postoperative gastroscopy was conducted after 5 months. (a) A reconstructed gastric fundus with a good shape. (b) A little erosion close to the anastomosis. 